\title{
SISTEMATIZAÇÃO DA ASSISTÊNCIA DE ENFERMAGEM PARA GESTANTES COM PRÉ ECLÂMPSIA E/OU ECLÂMPSIA: REVISÃO INTEGRATIVA DA LITERATURA
}

\author{
Mariana Antunes Carvalho de Souza ${ }^{1}$ \\ Maria Aparecida Xavier Moreira da Silva ${ }^{2}$
}

RESUMO: A Síndrome Hipertensiva Específica da Gestação (SHEG) se caracteriza como um distúrbio que promove intensas alterações fisiológicas na gestante podendo ocasionar a morte da mãe e do bebê. Esse estudo traz como objetivo principal descrever o papel do enfermeiro na elaboração da Sistematização da Assistência de Enfermagem e do Processo de Enfermagem para paciente gestante com pré-eclâmpsia e/ou eclâmpsia. Trata-se de uma pesquisa de revisão integrativa da literatura que foi realizada no período entre março à outubro de 2021. A amostra foi composta por 33 publicações. Os resultados apontam que as gestantes em pré-eclâmpsia e/ou eclâmpsia apresentam como necessidades de assistência de enfermagem as necessidades psicobiológicas, psicossociais e psicoespirituais. Mediante essas necessidades foram identificados os seguintes diagnósticos de enfermagem para atendimento dessas gestantes: Risco de função cardiovascular prejudicada, Risco de binômio mãe-feto perturbado, Risco de infecção, Risco de sangramento, Conforto prejudicado, Ansiedade, Dor aguda e Náusea. A pesquisa evidenciou que gestantes com eclâmpsia e/ou pré-eclâmpsia correm um grande risco de vir a óbito, não somente elas como também seus bebês. O papel do enfermeiro na aplicabilidade da SAE é de suma importância e que, quando realizada de forma adequada, a SAE permite ao enfermeiro a identificação precoce de riscos ou alterações que esta gestante esteja sofrendo, com indício de pré-eclâmpsia ou eclâmpsia, na qual pode se ter um desfecho favorável, evitando a ocorrência de óbito materno-fetal. Desta forma, a SAE pode instrumentalizar o enfermeiro para uma abordagem assistencial, direcionando cuidados específicos para essas pacientes, evitando-se resultados negativos.

Palavras-chave: Papel do Enfermeiro. Gestantes. Sistematização da Assistência de Enfermagem. Eclâmpsia. Pré-eclâmpsia.

\footnotetext{
${ }^{1}$ Graduanda do Curso de Enfermagem da Universidade de Mogi das Cruzes

${ }^{2}$ Mestre em Políticas Públicas pela Universidade de Mogi das Cruzes. Especialista em Administração Hospitalar pela Universidade de Ribeirão Preto. Graduada em Enfermagem pela Universidade de Mogi das Cruzes. Docente do Curso de Graduação em Enfermagem da Universidade de Mogi das Cruzes e Pós Graduação em Gestão em Saúde. Atuação nas disciplinas de Semiologia e Semiotécnica em Enfermagem, Administração em Enfermagem, Enfermagem em Home Care, Tópicos Avançados II e Enfermagem na Saúde da Criança e Adolescente. Membro do Comitê de Ética em Pesquisa (CEP) da Universidade de Mogi das Cruzes.
} 
ABSTRACT: Pregnancy-Specific Hypertensive Syndrome (SHEG) is characterized as a disorder that promotes intense physiological changes in pregnant women, which can lead to the death of mother and baby. This study has as its main objective to describe the role of nurses in the elaboration of the Systematization of Nursing Care and the Nursing Process for pregnant patients with pre-eclampsia and/or eclampsia. This is an integrative literature review research that was carried out between March and October 2021. The sample consisted of 33 publications. The results show that pregnant women in preeclampsia and/or eclampsia present psychobiological, psychosocial and psychospiritual needs as nursing care needs. Based on these needs, the following nursing diagnoses were identified for the care of these pregnant women: Risk of impaired cardiovascular function, Risk of disturbed mother-fetus binomial, Risk of infection, Risk of bleeding, Impaired comfort, Anxiety, Acute pain and Nausea. The research showed that pregnant women with eclampsia and/or pre-eclampsia are at high risk of death, not only they but also their babies. The role of the nurse in the applicability of the SAE is of paramount importance and that, when performed properly, the SAE allows the nurse to identify early risks or changes that this pregnant woman is suffering, with evidence of pre-eclampsia or eclampsia, in which it can have a favorable outcome, avoiding the occurrence of maternalfetal death. In this way, the SAE can equip nurses for a care approach, directing specific care for these patients, avoiding negative results.

Keywords: Role of the Nurse. Pregnant women. Systematization of Nursing Care. Eclampsia. Pre eclampsia.

\section{INTRODUÇÃO}

A Síndrome Hipertensiva Específica da Gestação (SHEG) é um distúrbio que tem elevadas chances de ocasionar a morte da mãe e do bebê, há uma grande quantidade de casos no nosso país, mantendo o primeiro lugar dentre as enfermidades derivadas da gravidez e também é a primeira patologia causadora do óbito maternal, essencialmente quando se agrava evoluindo para uma eclâmpsia e para a síndrome de HELLP "hemólise $(\mathrm{H})$, níveis elevados de enzimas hepáticas (EL) e contagem baixa de plaquetas (LP)”, atuando de forma prejudicial na gravidez. Desse modo, a assistência de enfermagem individual para essas gestantes é primordial para descoberta antecipada dessas patologias, a fim de ser feito as intervenções, possibilitando menos riscos na gestação (AGUIAR et al., 2010; PERAÇOLI et al., I998, p.165).

A pré-eclâmpsia, eclâmpsia e síndrome de HELLP são Síndromes Hipertensivas Específicas da Gestação. A Síndrome de HELLP é uma complicação da eclampsia (MELO 
et al, 2009). Para Ferreira et al. (2016, p. 325) a pré-eclâmpsia é uma alteração que pode afetar a gestante ocorrendo "após a vigésima semana gestacional, durante o parto e até 48 horas pós-parto" Tendo uma incidência entre 5 a $8 \%$ das gestações, sendo "uma condição que progride rapidamente, caracterizada por aumento tensional da pressão arterial (PA) e presença de proteinúria”.

Este estudo objetiva descrever o papel do enfermeiro na elaboração da Sistematização da Assistência de Enfermagem e do Processo de Enfermagem para paciente gestante com pré-eclâmpsia e/ou eclâmpsia; descrever o papel do enfermeiro na elaboração da Sistematização da Assistência de Enfermagem e do Processo de Enfermagem para paciente gestante com pré-eclâmpsia e/ou eclâmpsia; identificar os principais diagnósticos de enfermagem para paciente gestante com pré-eclâmpsia e/ou eclâmpsia a partir da taxonomia NANDA I e, identificar as necessidades de cuidados de paciente gestante com pré-eclâmpsia e/ou eclâmpsia.

\title{
REFERENCIAL TEÓRICO
}

\section{TEORIA DAS NECESSIDADES HUMANAS BÁSICAS}

O termo 'Necessidades humanas básicas' é definido por Horta (1979, p. 39) como sendo um conjunto de "estados de tensões, conscientes ou inconscientes, resultantes dos desequilíbrios homeodinâmicos dos fenômenos vitais. [...] que o indivíduo, família e comunidade apresentam decorrentes do desequilíbrio de suas necessidades básicas que exijam uma resolução" e que essas necessidades estão divididas em três níveis: necessidades psicobiológicas, necessidades psicossociais e necessidades psicoespirituais.

\begin{abstract}
As necessidades psicobiológicas concentram: oxigenação; hidratação; eliminação; sono e repouso; nutrição; exercício e atividades físicas; abrigo; mecânica corporal; motilidade; sexualidade, cuidado corporal; integridade cutâneo-mucosa e física; regulação térmica, hormonal, neurológica, hidroeletrolítica, imunológica, crescimento celular, vascular; percepção dos órgãos do sentido; ambiente; terapêutica e locomoção. As necessidades psicossociais: segurança, amor, liberdade, comunicação, criatividade, aprendizagem, gregária, recreação, lazer, espaço, orientação no tempo e espaço, aceitação, auto realização autoestima, participação, autoimagem e atenção. As Necessidades psicoespirituais: religiosa ou teológica, ética e de filosofia de vida (HORTA, 1979, p.40).
\end{abstract}

Problemas de enfermagem são quaisquer circunstâncias e/ou condições que surgem 
através de indivíduos, famílias ou comunidades que precisam de ajuda profissional. São solucionados através de toda a SAE desenvolvida, após a identificação dos problemas através do Histórico, acontece a análise dos problemas para identificação das necessidades e dependências de enfermagem pelo paciente e elencar os Diagnóstico de enfermagem para assim desenvolver os cuidados necessários para a melhora desse problema (HORTA, 1979).

A Teoria das Necessidades Humanas Básicas são subtendidas, como sistemas globais, essenciais que, correlacionadas subsidiam a prática do cuidado, As necessidades humanas básicas são universais e estão inter-relacionadas, mas cada pessoa manifesta de formas diferentes, dependendo de vários fatores da vida e situação (HORTA, 1979).

Para Horta (1979, p. 40) uma necessidade, quando manifestada, é identificada por meio de sinais e sintomas, os quais se caracterizam como problemas de enfermagem, desta forma, consideram-se problemas de enfermagem os sinais e sintomas como: "cianose, dispneia, respiração ortopédica, lentidão, cansaço, fadiga, insegurança, agitação, irritabilidade, ansiedade, medo, euforia, tontura, coriza, tosse, sangramento, tabagismo, obstrução das vias aéreas, estase circulatória, mudança no ritmo e frequência dos movimentos respiratórios etc.

Ainda no campo de necessidades, o amor, como necessidade humana é um "processo dinâmico de troca de energia emocional positiva entre os seres vivos". No entendimento dessa necessidade, os sentimentos e sensações que podem identificá-la são: “ansiedade, insegurança, tensão, rejeição, negatividade, apatia, depressão, solidão, fuga, medo, dor, agressão, anorexia, perda de peso, dependência, obesidade, insônia, choro, indiferença, colapso, euforia, exibicionismo, crime, comportamento desviante, etc" (HORTA, 1979, p.4I).

\section{SISTEMATIZAÇÃO DA ASSISTÊNCIA DE ENFERMAGEM (SAE) E PROCESSO DE ENFERMAGEM}

A SAE é amparada pela Resolução COFEN no 358/2009 que estabelece a obrigatoriedade da implantação da SAE e a implementação do Processo de Enfermagem 
(PE) em todas as instituições de saúde, sejam elas instituições privadas ou públicas, desde que haja a realização da prática de enfermagem. Machado et al. (2019) afirmam que a SAE é uma metodologia baseada em ciência que contribui para uma prática de enfermagem profissional:

Corroborando Mola et al. (2019) descrevem que a SAE organiza a prática de enfermagem e o fluxo da assistência, oferecendo subsídios para o desenvolvimento metodológico de práticas interdisciplinares e humanizadas de cuidado. A SAE estipula o tipo de profissional requerido, as técnicas, os procedimentos, os métodos, os objetivos e os recursos materiais para a produção do cuidado, além de definir a natureza e o tipo do trabalho a ser realizado.

Segundo a Resolução COFEN-358/2009, o Processo de Enfermagem é composto por cinco etapas nas quais estão interligadas. A coleta de dados é a primeira etapa do PE e consiste em duas fases a 'entrevista' com o paciente obtendo informações sobre o histórico da família e precedentes de doenças e, o 'exame' físico para a detecção de alterações, sinais e sintomas que estejam relacionados ao problema de saúde-doença. O Diagnóstico de Enfermagem, segunda etapa do $\mathrm{PE}$, é realizado para a interpretação e julgamento clínico de enfermagem sobre os dados obtidos na etapa anterior, sendo elencados os diagnósticos que mais tem a ver com as respostas do paciente (COFEN, 2009, s.p.).

A partir da escolha pelos diagnósticos de enfermagem, o enfermeiro realizará a terceira etapa do PE que é o Planejamento de Enfermagem. Nessa etapa são determinados os resultados que se pretende alcançar com o paciente e as intervenções que irão ser realizadas para o alcance desses resultados. A quarta etapa, caracterizada como Implementação de Enfermagem se firma na execução das ações listadas no processo anterior e, por último, a quinta etapa denominada Avaliação de Enfermagem é a etapa que mostra a evolução do paciente, os resultados que foram alcançados de acordo com os cuidados realizados e subsidia a elaboração de um novo ciclo de PE, tornando-se um processo constante, dinâmico e resolutivo para as necessidades de assistência de enfermagem (COFEN, 2009). 


\section{TAXONOMIA NANDA-I}

Taxonomia é uma forma de classificar ou ordenar coisas em categorias; é um esquema de classificação hierárquica de grupos principais, subgrupos e itens (NANDA-I, 2018-2020)

No início dos anos 1970, enfermeiras e educadores americanos verificaram que a enfermeira diagnosticou de forma independente e tratou "coisas" (referente aos pacientes e suas famílias) de forma diferente dos diagnósticos médicos. Esta descoberta abriu uma nova porta para a taxonomia desses Diagnósticos e criação de organizações profissionais que atualmente é denominado NANDA Internacional (NANDA-I). Taxonomia possui i3 domínios, com 47 classes no total e 244 diagnósticos, 17 deles são novos (NANDA-I, 20182020).

\section{TAXONOMIA NOC}

Contém 385 resultados. Cada resultado inclui um título, uma definição e um conjunto de indicadores. Os resultados ajudam enfermeiros e outros profissionais de saúde a avaliar e quantificar a condição de pacientes, cuidadores, famílias ou comunidades. $\mathrm{O}$ enfermeiro pode quantificar as mudanças no estado do paciente após a intervenção e monitorar seu progresso. Fornece orientações práticas sobre como usar o NOC na prática clínica. O Capítulo I revisa a pesquisa de cuidados de saúde e resultados de enfermagem e a pesquisa preliminar para desenvolver e refinar essa classificação. Este capítulo é útil para enfermeiras que estão começando a considerar o uso de medição de resultados na prática e desejam revisar o uso de resultados na enfermagem. O Capítulo 2 descreve as classificações atuais nesta edição. As definições de termos, perguntas frequentes e notícias são destacadas. O Capítulo 3 discute como usar o NOC na prática clínica, faz sugestões úteis e fornece exemplos atualizados de como usar o NOC em várias situações. O Capítulo 4 discute o uso de NOC em educação e pesquisa e enfatiza o trabalho atual e as perspectivas futuras (NOC, 2008). 

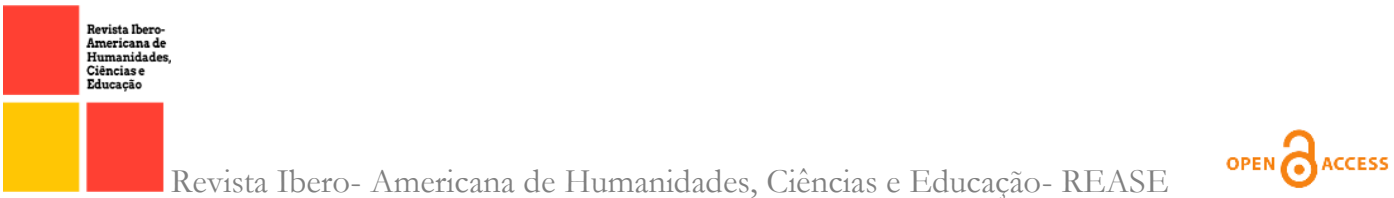

\section{Taxonomia NIC}

A equipe NIC foi criada em 1987. A primeira edição foi publicada em 1992. NIC é uma linguagem padronizada abrangente para descrever tratamentos realizados por enfermeiros. Três capítulos foram atualizados para apresentar a classificação: o Capítulo I analisa a NIC e cobre 20 questões relacionadas à NIC que ás vezes surgem; o Capítulo 2 descreve o início da NIC em 1987 e propõe a necessidade de criar NIC e, em seguida, descreve a pesquisa conduzida para seus desenvolvimento e manutenção; o Capítulo 3 enfoca a implementação e o uso da NIC na prática e no ensino. Há um total de 7 domínios, 30 classes e 542 intervenções nesta edição (NIC, 2010).

\section{MÉTODO}

Trata-se de uma pesquisa de revisão integrativa da literatura que foi realizada por meio da BVS (Biblioteca Virtual em Saúde) para busca de artigos nas bases de dados da LILACS (Literatura Latino-americana e do Caribe em Ciências da Saúde) e BDENF

(Banco de Dados em Enfermagem) que tratem sobre a temática das necessidades, cuidados e papel do enfermeiro no atendimento de pacientes com eclâmpsia e pré-eclâmpsia. A revisão integrativa é a mais abrangente relacionada às revisões, acatando a inserção de estudos experimentais e não experimentais com o intuito de um entendimento geral do que foi analisado, associa também dados da literatura teórica e metódicas, além de incluir uma ampla variedade de propósitos. (SOUZA et al., 2010).

Para a coleta de dados foi utilizado o instrumento elaborado e validado por Ursi (2005) composto por 5 campos para a descrição dos dados encontrados: A) Identificação; B) Instituição sede do estudo; C) Tipo de publicação; D) Características metodológicas do estudo; E) Avaliação do rigor metodológico. O instrumento de Ursi (2005) foi transcrito para uma planilha da ferramenta Excel ${ }^{\circledR}$ para facilitar a organização dos dados coletados.

Como critério de inclusão optou-se por publicações no formato de artigos, livros e teses, publicados no período entre 2016 à 2021, no idioma português, com texto completo nas bases de dados, com delineamentos de pesquisa de campo, relatos de experiência e estudos de caso, tendo como assuntos principais: cuidados de enfermagem, gestantes de 
alto risco, sistematização da assistência de enfermagem, processo de enfermagem, papel do enfermeiro e diagnósticos de enfermagem. Foram excluídas publicações duplicadas nas bases de dados, com abordagem de assuntos gerais fugindo do objetivo desse estudo e publicações com acesso pago.

A coleta de dados foi realizada primeiramente na base de dados da LILACS (Literatura Latino-Americana e Caribe em Ciências da Saúde) e em seguida na BDENF (Banco de Dados em Enfermagem). Os descritores em saúde foram testados no DeCS (Descritores em Ciência da Saúde) e utilizados aos pares, sendo eles: gestantes and assistência de enfermagem, gestantes and eclâmpsia, papel do enfermeiro and Sistematização da Assistência de Enfermagem e, papel do enfermeiro and Processo de Enfermagem. Os dados coletados foram organizados em planilhas do Excel, primeiramente com o preenchimento do instrumento de Ursi (2005) para caracterização da amostra e na sequência foi realizado o fichamento das publicações que compõem a amostra por meio da leitura sistemática, onde se obteve as informações necessárias para a organização das categorias de análise.

Após leitura sistemática, os dados foram organizados e categorizados, onde emergiram três categorias de análise, sendo elas: I- Necessidades de Assistência de Enfermagem; 2- Diagnósticos de Enfermagem e, 3- Papel do Enfermeiro na elaboração da SAE e PE e, a partir da definição dessas categorias, os resultados foram analisados com base nos referenciais teóricos: Processo de Enfermagem de Wanda de Aguiar Horta, Resolução COFEN 358/2009 e Taxonomias NANDA-I, NIC e NOC.

\section{RESULTADOS}

A coleta de dados foi realizada no período entre março à outubro de 2021 e, de acordo com o que se apresenta no Quadro I, com o cruzamento dos descritores foram encontradas 1.252 publicações e com a utilização dos critérios de inclusão/exclusão foram evidenciados 416 estudos, sendo que 179 estudos estavam indexados na base de dados da LILACS e 237 estudos na base de dados da BDENF. 
Quadro I - Caracterização da coleta de dados a partir do cruzamento dos descritores em saúde nas bases de dados e critérios de inclusão/exclusão, Mogi das Cruzes, 202I.

\begin{tabular}{|c|c|c|c|c|c|c|c|}
\hline Descritores & $\begin{array}{c}\text { Base de } \\
\text { dados }\end{array}$ & $\begin{array}{l}\text { Estudos } \\
\text { encontrad } \\
\text { os }\end{array}$ & $\begin{array}{l}\text { Critério } \\
\text { s de } \\
\text { Inclusã } \\
\text { o }\end{array}$ & $\begin{array}{l}\text { Estudos } \\
\text { duplicado } \\
\text { s na } 2^{-} \\
\text {base }\end{array}$ & $\begin{array}{c}\text { Leitura } \\
\text { flutuant } \\
\text { e }\end{array}$ & $\begin{array}{c}\text { Leitura } \\
\text { sistemátic } \\
\text { a }\end{array}$ & $\begin{array}{l}\text { Seleçã } \\
\text { o para } \\
\text { a } \\
\text { amostr } \\
\quad \text { a }\end{array}$ \\
\hline \multirow{2}{*}{$\begin{array}{c}\text { Gestante } \\
\text { and } \\
\text { Assistência } \\
\text { de } \\
\text { Enfermagem }\end{array}$} & $\begin{array}{l}\text { LILAC } \\
\mathrm{S}\end{array}$ & 113 & 37 & -..- & I4 & 13 & 13 \\
\hline & $\begin{array}{l}\text { BDEN } \\
\mathrm{F}\end{array}$ & 132 & 49 & I8 & 8 & 6 & 5 \\
\hline \multirow{2}{*}{$\begin{array}{c}\text { Gestante } \\
\text { and } \\
\text { Eclâmpsia }\end{array}$} & $\begin{array}{l}\text { LILAC } \\
\mathrm{S}\end{array}$ & 42 & I2 & $-\cdots$ & 6 & 5 & 4 \\
\hline & $\begin{array}{l}\text { BDEN } \\
F\end{array}$ & I8 & I6 & 8 & 3 & o & o \\
\hline \multirow{2}{*}{$\begin{array}{c}\text { Papel do } \\
\text { Enfermeiro } \\
\text { and } \\
\text { Sistematizaç } \\
\text { ão da } \\
\text { Assistência } \\
\text { de } \\
\text { Enfermagem }\end{array}$} & $\begin{array}{l}\text { LILAC } \\
\mathrm{S}\end{array}$ & 314 & 66 & -..- & I5 & 9 & 6 \\
\hline & $\begin{array}{l}\text { BDEN } \\
\text { F }\end{array}$ & 334 & 86 & 22 & II & 5 & 3 \\
\hline \multirow{2}{*}{$\begin{array}{l}\text { Papel do } \\
\text { Enfermeiro } \\
\text { and Processo } \\
\text { de } \\
\text { Enfermagem }\end{array}$} & $\begin{array}{l}\text { LILAC } \\
\text { S }\end{array}$ & I4I & 64 & $\ldots$ & 3 & 3 & 2 \\
\hline & $\begin{array}{l}\text { BDEN } \\
\text { F }\end{array}$ & 158 & 86 & I5 & I & o & o \\
\hline \multicolumn{2}{|c|}{ TOTAL } & 1252 & 416 & 63 & 6I & $4 \mathrm{I}$ & 33 \\
\hline
\end{tabular}

Fonte: dados de pesquisa organizado pela pesquisadora. 
Quadro I - Caracterização da amostra $(\mathrm{n}=33)$ de acordo com o cruzamento dos descritores nas bases de dados LILACS e BDENF, Mogi das Cruzes, 202I.

\begin{tabular}{|c|c|c|c|c|}
\hline \multicolumn{5}{|c|}{ I Cruzamento dos descritores: Gestantes and Assistência de Enfermagem } \\
\hline oI & LILACS & $\begin{array}{l}\text { Tecnologias não invasivas na assistência às parturientes de alto risco: } \\
\text { percepções de enfermeiras obstétricas. }\end{array}$ & ARES et al. & 2021 \\
\hline 02 & LILACS & Assistência humanizada no pré-natal de alto risco: percepções de enfermeiros. & JORGE et al. & 2020 \\
\hline 03 & LILACS & $\begin{array}{l}\text { Enfermagem e gestantes de alto risco hospitalizadas: desafios para } \\
\text { integralidade do cuidado. }\end{array}$ & SOUZA et al. & 2020 \\
\hline 05 & LILACS & Diagnósticos de Enfermagem mais prevalentes em gestantes de alto risco. & TELES et al. & 2019 \\
\hline o6 & LILACS & $\begin{array}{l}\text { Processo assistencial das mulheres com morbidade materna grave: um estudo } \\
\text { misto. }\end{array}$ & VILLALBA & 2019 \\
\hline 07 & LILACS & Grupo de gestantes de alto-risco como estratégia de educação em saúde. & ALVES et al. & 2019 \\
\hline o8 & LILACS & $\begin{array}{l}\text { Validação de instrumento para histórico de enfermagem materno-infantil } \\
\text { utilizando Horta: estudo metodológico. }\end{array}$ & ALMEIDA et al. & 2018 \\
\hline $\mathrm{I} 2$ & LILACS & $\begin{array}{l}\text { Avaliando diagnósticos e intervenções de enfermagem no trabalho de parto e na } \\
\text { gestação de risco. }\end{array}$ & MEDEIROS et al. & 2016 \\
\hline
\end{tabular}




\begin{tabular}{|c|c|c|c|c|}
\hline 13 & LILACS & Processo de enfermagem em centro obstétrico: perspectiva dos enfermeiros & FRAGA et al. & 2018 \\
\hline I4 & BDENF & Construção e validação de um histórico de enfermagem para consulta pré-natal. & TAVARES et al. & 2020 \\
\hline 16 & BDENF & $\begin{array}{l}\text { Cuidado hospitalar de mulheres que vivenciaram a gestação de alto risco: } \\
\text { contribuições para a enfermagem. }\end{array}$ & MELO & 2016 \\
\hline I7 & BDENF & Assistência de enfermagem a parturientes acometidas por pré-eclâmpsia. & OLIVEIRA et al. & 2016 \\
\hline $\mathrm{I} 8$ & BDENF & Perfil de gestantes cardiopatas: alto risco & FELCZAK et al. & 2018 \\
\hline \multicolumn{5}{|c|}{ 2- Cruzamento dos descritores: Gestantes and Eclâmpsia } \\
\hline 20 & LILACS & $\begin{array}{l}\text { Assistência de enfermeiros na síndrome hipertensiva gestacional em hospital } \\
\text { de baixo risco obstétrico. }\end{array}$ & OLIVEIRA et al. & 2017 \\
\hline $2 \mathrm{I}$ & LILACS & $\begin{array}{l}\text { A comunicação de más notícias pelo enfermeiro no cenário do cuidado } \\
\text { obstétrico. }\end{array}$ & ROCHA et al. & 2016 \\
\hline 22 & LILACS & Crise hipertensiva gestacional & POZZA et al. & 2016 \\
\hline \multicolumn{5}{|c|}{ 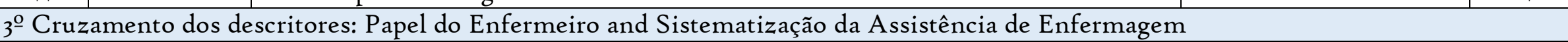 } \\
\hline 25 & LILACS & Pré-eclâmpsia/eclâmpsia. & PERAÇOLI et al. & 2019 \\
\hline 26 & LILACS & Características maternas e fatores de risco para pré-eclâmpsia em gestantes. & FERREIRA et al. & 2019 \\
\hline \multicolumn{5}{|c|}{$3^{\circ}$ Cruzamento dos descritores: Papel do Enfermeiro and Sistematização da Assistência de Enfermagem (continuação) } \\
\hline 27 & LILACS & $\begin{array}{l}\text { Cuidados pré-natais e puerperais às gestantes de um centro de saúde de Minas } \\
\text { Gerais quanto ao risco de pré-eclâmpsia: aspectos clínicos, nutricionais e }\end{array}$ & SILVA et al. & 2017 \\
\hline
\end{tabular}




\begin{tabular}{|c|c|l|l|c|c|}
\hline & & \multicolumn{2}{l|}{ terapêuticos. } & \\
\hline 28 & LILACS & Síndrome hipertensiva e resultados perinatais em gestação de alto risco. & ANTUNES et al. & 20I7 \\
\hline 29 & BDENF & $\begin{array}{l}\text { Aspectos sociodemográfico, clínico-obstétrico e laboratorial na síndrome } \\
\text { hipertensiva na gravidez. }\end{array}$ & GONÇALVES et al. & 20I9 \\
\hline 30 & BDENF & $\begin{array}{l}\text { Medidas preventivas das síndromes hipertensivas da gravidez na atenção } \\
\text { primária. }\end{array}$ & THULER et al. \\
\hline 31 & BDENF & $\begin{array}{l}\text { Perfil de gestantes com síndrome hipertensiva em uma maternidade pública. } \\
\text { Cruzamento dos descritores: Papel do Enfermeiro and Processo de Enfermagem }\end{array}$ & 20I7 \\
\hline 32 & LILACS & $\begin{array}{l}\text { Atitudes dos enfermeiros frente ao Processo de Enfermagem de um hospital } \\
\text { público: estudo descritivo }\end{array}$ & DIAS e DURAN \\
\hline 33 & LILACS & $\begin{array}{l}\text { Compreensões e desafios acerca da sistematização da assistência de } \\
\text { enfermagem. }\end{array}$ & CASTRO et al. & 20I6 \\
\hline
\end{tabular}

Fonte: dados de pesquisa organizado pela pesquisadora. 
Do total de estudos encontrados $(n=416)$, foram excluídos da base de dados da BDENF 63 estudos, pois, estavam duplicados e, a partir da leitura dos títulos dos estudos resultantes $(n=353)$ foram selecionados para leitura flutuante 6r estudos, sendo 38 da LILACS e 23 da BDENF. Da leitura flutuante foram escolhidos 4I artigos para a realização da leitura sistemática, a qual conduziu para a construção da amostra deste estudo $(n=33)$. Os demais estudos foram excluídos por não atender às questões norteadoras e os objetivos desse estudo. A maioria dos estudos $(n=25)$ foi encontrada na base de dados da LILACS e as demais $(\mathrm{n}=8)$ na base de dados da BDENF, conforme apresentado no Quadror.

No Quadro 2 estão apresentadas as informações sobre as bases de dados, títulos das publicações, nomes dos autores e ano de publicação de acordo com o cruzamento dos descritores.

A partir dessa organização foi possível a realização do agrupamento dos dados para a composição das categorias de análise: I) Necessidades de Assistência de Enfermagem e suas subcategorias: a) Necessidades Psicobiológicas, b) Necessidades Psicossociais e, c) Necessidades Psicoespirituais; 2) Diagnósticos de Enfermagem e, 3) Papel do Enfermeiro na elaboração da SAE e PE.

\section{NECESSIDADES DE ASSISTÊNCIA DE ENFERMAGEM}

Esta primeira categoria de análise apresenta as principais necessidades de assistência e/ou cuidados de enfermagem de gestantes com pré-eclâmpsia ou eclâmpsia apontadas pelos estudos e, para melhor entendimento, está organizada e dividida em subcategorias: a) Necessidades Psicobiológicas, b) Necessidades Psicossociais e, c) Necessidades Psicoespirituais.

Considerando as necessidades da psicobiologia, psicologia social e espiritual, enquanto registra o histórico de enfermagem na consulta pré-natal significa ir além do modelo biomédico para obter uma compreensão mais profunda da saúde e do potencial de vida do indivíduo (TAVARES et al., 2019). 


\section{a) Necessidades Psicobiológicas}

Para Aldrighi et al. (2021) a gestação em mulheres idade igual ou maior que 35 anos é considerada de risco, pois, é comum a partir dessa idade desenvolver doenças crônicas tendo a tendência para o desenvolvimento de síndromes hipertensivas gestacional, destacando-se entre elas a pré-eclâmpsia e a eclâmpsia, colocando em risco a saúde dessa mulher.

Segundo a Sociedade Brasileira de Pediatria (SBP), a síndrome hipertensiva materna é um risco e precisa de intervenções imediatas. Pesquisas indicam nas suas conclusões a precisão e importância de fazer investigações que fale sobre os resultados perinatais perante as especificidades e complexidade diagnóstica da Pré-Eclâmpsia Grave (CASSIANO et al., 2019).

Para diagnóstico diferencial entre as emergências hipertensivas (principalmente pré-eclâmpsia) no momento da admissão, devemos realizar uma avaliação inicial que inclui: $\mathrm{P} / \mathrm{C}$ (proteinúria/creatinúria) e reavaliação da PA (Pressão Arterial). Caso persistência de aumento dos níveis tensionais, sinais de comprometimento sistêmico ou mal estar fetal, está indicado internação hospitalar seguido de exames laboratoriais complementares, como: hemograma e plaquetas, TGO (Aspartato Amiotransferase), TGP (Alanina Amnotransferase), LDH (Lactato Desidrogenase), creatinina, ácido úrico e proteinúria de 24horas (POZZA et al., 2016, p.3).

Ao receber diagnóstico da pré-eclâmpsia, a prioridade é ter a estabilização clínica fazendo a prevenção do óbito materno e fetal, através: de orientações sobre os sinais de comprometimento da doença, de encaminhamento e assistência em serviços terciários e com assistência neonatal qualificada, do bom controle pressórico, da prevenção da eclâmpsia ou de sua recorrência e na identificação precoce de alterações laboratoriais, principalmente aquelas relacionadas à síndrome HELLP e a avaliação do bem-estar fetal (PERAÇOLLI et al., 2019).

Para Ares et al. (202I, p. 2) a prevenção das principais causas de morte em gestantes está na estrutura da assistência qualificada e nos acessos aos serviços de saúde para atendimento de "pré-natal, da classificação de risco, da vinculação, da assistência 
multiprofissional e interdisciplinar nos diferentes níveis de complexidade, com práticas clínicas compartilhadas e baseadas em evidências científicas e da adoção das boas práticas obstétricas".

Uma providência a ser tomada para que se evite que a pré-eclâmpsia se torne algo mais grave é um pré-natal bem feito tendo um bom acompanhamento, no qual destaca o papel do enfermeiro visto a assistência a gestantes com pré-eclâmpsia e a percepção precoce de intercorrências obstétricas, diminuindo a chances do óbito materno-fetal (FERREIRA et al., 2019). É realmente necessário que a enfermagem preste uma boa assistência principalmente no pré-natal, identificando antecipadamente alguma intercorrência materno-fetal, fazendo a monitorização da pressão arterial, e fazer conscientização caso mulheres que já tenham hipertensão queiram engravidar é bom se planejar antes para que não ocorra nenhum agravo na gestação (ANTUNES et al., 2017).

Para reduzir a chances de aparecimento e evolução da pré-eclâmpsia é proposto algumas intervenções como: o uso de ácido acetilsalicílico (AAS) e a suplementação de cálcio (PERAÇOLLI et al., 2019). Além da terapêutica medicamentosa, também devem ser realizadas ações de educação em saúde, orientações para observação das alterações fisiológicas durante a gestação, métodos não medicamentosos para o alívio da dor do parto, crescimento e desenvolvimento, e desenvolvimento fetal e amamentação. As práticas acima ajudam as mulheres a compreender os fatores de risco, complicações na gravidez, saúde materna e neonatal, reduzindo assim o medo do parto (JORGE et al., 2020).

Ressalta-se a relevância das intervenções durante o pré-natal de alto risco, pois essas intervenções podem prevenir e tratar doenças que afetam a mãe e o feto. A equipe de enfermagem deve tratar bem e atenciosamente as gestantes, apresentando uma proximidade do cuidado de enfermagem medindo a pressão arterial, aplicando insulina, dando banho, ajudando na amamentação e na hora do parto (JUNIOR et al., 2017; MELO et al., 2016).

Quando a eclâmpsia aparece no final da gravidez, deve ser antecipado o parto da cliente identificando a origem da pré-eclâmpsia, antes que iniciem as convulsões. Quando a eclâmpsia surge no momento do parto, ele é antecipado, e após o feto sair ou vir a óbito 
ocorre a melhora da parturiente, entre 12 a 24 horas. Já a eclâmpsia ocorrida no pós-parto, as convulsões se iniciam em seguida ao parto, normalmente nas primeiras 24 horas (NOBREGA et al., 2016).

A dieta de mulheres grávidas deve ser monitorada quanto à nutrição, levando em consideração a quantidade de alimentos ingeridos e refeições permitidas, restrições alimentares, doenças subjacentes, ganho de peso normal permitido, especialmente a avaliação do índice de massa corporal (SILVA et al., 2017). Isso mostra que, na prática de enfermagem de gestantes SHEG (síndrome hipertensiva específica da gravidez), o monitoramento do débito urinário e da frequência respiratória, a manutenção da curva de pressão e o monitoramento da vitalidade fetal por meio da ausculta de frequência cardíaca são intervenções positivas e medidas valiosas para o alcance da qualidade (BELARMINO et al., 2018).

O parto é a única maneira eficaz de tratar a pré-eclâmpsia. O parto vaginal é mais adequado para mulheres com pré-eclâmpsia e eclâmpsia para evitar o estresse. Esta é uma operação realizada em organismos que mudaram de maneiras diferentes. Pacientes com

SHG (Síndrome Hipertensiva da Gestação) têm parâmetros clínicos e laboratoriais mais graves, maiores taxas de cesárea, piores resultados maternos e perinatais, porque múltiplos órgãos estão envolvidos, é necessário monitoramento rigoroso durante a gravidez, o que leva a resultados de exames laboratoriais alterados (SILVA et al., 2020; GONÇALVES et al., 2019).

\section{b) Necessidades Psicossociais}

Aldrighi et al. (202I) descrevem que os aspectos sociodemográficos como escolaridade e padrão socioeconômico, quando associados, podem interferir no entendimento dessas mulheres quanto ao seu processo de saúde-doença, comprometendo a manutenção do acompanhamento e tratamento para prevenção de riscos na gestação. Os autores salientam a importância do enfermeiro na coleta de dados que possam evidenciar o contexto da saúde de cada gestante, principalmente na evidência de situações que possam indicar complicações presentes ou estejam em risco de acontecer. 
Corroborando, Villalba (2019, p. 59) relata que a "baixa condição socioeconômica materna" é fator predisponente para o "desenvolvimento de morbidade materna grave nas gestantes”, pois, a falta de condições socioeconômicas pode fazer com que as gestantes posterguem á procura de unidades de saúde para a realização do pré-natal, tornando-se vulneráveis às complicações gestacionais.

Villalba (2019) salienta que uma das necessidades das gestantes de alto risco é a acessibilidade ao sistema de saúde, destacando-se a escassez de unidades de saúde que atendam esse perfil de clientela. Tal situação promove o deslocamento da gestante para a procura de serviços não especializados, comprometendo ainda mais a sua situação de saúde.

Por outro lado, Ares et al. (202I) retratam que a gestação por si só acarreta inúmeras alterações no corpo da gestante, entretanto, uma gestação de alto risco além das alterações fisiológicas, desenvolve alterações biopsicossociais apresentando sintomas como angústias, ansiedade e medos e, Borba et al. (2019) reforçam que as transformações que ocorrem no corpo da gestante, por menor que sejam, produzem as alterações biopsicossociais citadas

por Ares et al. (202I), demandando de uma atenção por parte dos profissionais de saúde, enfermeiros e médicos, para melhor atendimento dessa gestante para prevenção de maiores complicações.

O papel do enfermeiro no trabalho multidisciplinar em saúde inclui avaliação psicossocial e nutricional, educação em saúde, consulta perinatal, gestão de serviços e apoio à decisão, visando identificar os riscos (JORGE et al., 2020).

As condições para categorizar os riscos da gravidez envolvem condições clinicas obstétricas e aspectos psicoemocionais, com o objetivo de compreender os riscos potenciais e requer adaptação física e psicológica e atenção especial. Nesse processo de enfermagem, o enfermeiro se destaca e pode identificar fatores que interferem no processo de educação, portando, a enfermagem desempenha um papel importante no cotidiano de trabalho dessas mulheres, cabendo-lhes a responsabilidade de orientar, assistir e apoiar, e o uso de uma linguagem simples pode favorecer o processo psicoemocional (ALVES et al., 2019). 
O profissional enfermeiro também orienta sobre o parto normal, amamentação e puerpério, e se empenha constantemente para evitar interferências no processo de saúde e doença das usuárias do sistema durante a gestação (JUNIOR et al., 2017).

Para Melo et al. (2016), a equipe de enfermagem é sensível à escuta e ao cuidado da gestante, e presta igual atenção à realização de procedimentos técnicos indispensáveis à saúde da mãe e do bebê, bem como à subjetividade ao longo de toda a gestação e parto. Essa dupla avaliação fornece o ponto de partida necessário para obter orientação a partir das dúvidas da mulher, permitindo impor uma conexão mais humana através de vínculo confiança, tendo a enfermagem como uma arte mediada pelo conhecimento cientifico e, de acordo com Nobrega et al. (2016) as parturientes devem receber orientação em relação a possíveis problemas e sinais antecipados de eclampsia na alta hospitalar (NOBREGA et al., 2016).

É urgente a importância de uma equipe preparada para cuidar da portadora desta doença, a presença de uma enfermeira é fundamental, que deve acolher e acompanhar o paciente com hipertensão de forma digna e humana durante todo o processo do parto, fornecendo um suporte emocional, porque este é um período de extrema ansiedade e medo (OLIVEIRA et al., 2017).

Corroborando, Rocha et al. (2016), ressaltam que essas etapas requerem o suporte emocional e teórico do enfermeiro e, portanto, podem ser utilizadas como um indicador do momento que o paciente está vivenciando, alcançando a melhor forma de abordá-lo e buscando a sintonia com o processo pelo qual ele e sua família estão passando. Nesse mesmo entendimento, Souza et al. (2020) descrevem que as gestantes necessitam de uma assistência de enfermagem na qual seja compreensiva e tenha uma boa interpretação para suprir as necessidades da paciente, precisa também de apoio emocional com monitoramento do risco gestacional. Esse manejo, de acordo com Thuler et al. (2018), pode ser difícil quando a prevenção da hipertensão, induzida pela gravidez, se concentra apenas na redução do ganho de peso, pois também requer exercícios para realizar a educação materna. 


\section{c) Necessidades Psicoespirituais}

A fé é um sentimento bem relatado pelo autor. Vale destacar que as características dos colaboradores são a universalidade e a persistência diante das dificuldades da época. A fé, para essas mulheres, pode ser a explicação e a motivação para caminhar em direção ao seu objetivo: ser mãe e ter seus filhos o mais rápido possível (LIMA et al., 2016).

Enfatizar a soma de esforços para reduzir a morbimortalidade materna, perinatal e neonatal, e priorizar a obstetrícia e o recém-nascido no parto e puerpério sob a ótica da ética e do cuidado à gestante e da ética e cuidado dos profissionais de saúde que acolhem a gestante Humanização da assistência à criança. E implementar estratégias benéficas de saúde no monitoramento do parto, comprometendo assim as intervenções comumente usadas, o que cria mais riscos do que benefícios (MELO et al., 2016).

A verdade é a base de um relacionamento de confiança. Portanto, com base nos princípios da bioética, pode-se dizer que comunicar a verdade do diagnóstico aos pacientes e seus familiares é um grande benefício para eles, pois possibilita que ambas as partes participem ativamente do processo de tomada de decisão (ROCHA et al., 2016).

\section{DIAGNÓSTICOS DE ENFERMAGEM PARA GESTANTES COM PRÉ- ECLÂMPSIA OU ECLÂMPSIA}

A aplicação do PE (Processo de Enfermagem) vem contribuindo para o desenvolvimento de sistemas de classificação de enfermagem para os elementos da prática profissional, como a taxonomia e diagnóstico de enfermagem da NANDA- I, a Classificação de Intervenções de Enfermagem, a Classificação de Resultados de Enfermagem, o Sistema Omaha, a Classificação dos Cuidados Domiciliares de Saúde e a Classificação Internacional para a Prática da Enfermagem. Até 2002, NANDA era uma sigla para American Nursing Diagnostics Association. No entanto, esse não é mais o nome da organização. Em 2002, considerando o crescimento significativo da associação fora da América do Norte, ela mudou oficialmente para NANDA International (CASTRO et al., 2016). 
O PE é uma exigência do Conselho Federal de Enfermagem (COFEN) na prática de enfermagem, exigindo que os serviços de enfermagem invistam na construção e verificação de instrumentos que atendam às suas particularidades para nortear e padronizar o funcionamento do processo (ALMEIDA et al., 2018).

O exame físico e a anamnese são a base para subsidiar a próxima etapa do $\mathrm{PE}$, a do diagnóstico de enfermagem. Além de subsidiar a reflexão e avaliação da prática profissional de enfermagem, pode melhorar a qualidade, visibilidade e nível de assistência de enfermagem (ALMEIDA et al., 2018).

No que se refere à melhoria da qualidade da assistência prestada, a clientela materno-infantil merece atenção especial, pois o Brasil possui sérias causas evitáveis de indicadores de mortalidade materna. Para reverter essa situação, a Organização das Nações Unidas (ONU) definiu os Objetivos de Desenvolvimento do Milênio há I8 anos que ainda não foram totalmente alcançados, incluindo aqueles relacionados à promoção da saúde materna e neonatal. Então, em 2015, as Nações Unidas definiram novas prioridades, chamadas de Objetivos de Desenvolvimento Sustentável (ODS) (ALMEIDA et al., 2018).

Uma das metas da terceira meta de desenvolvimento sustentável é reduzir a mortalidade materna global e acabar com as mortes neonatais evitáveis por meio de ações que melhorem a qualidade da atenção pré-natal, parto e pós-natal. Partindo dessa hipótese, para produzir uma assistência humanizada e de qualidade na atenção à saúde maternoinfantil, é necessário organizar o trabalho para que o enfermeiro possa compreender o perfil da clientela que atende e as necessidades humanas e sociais afetadas, sejam elas estão em pré-natal, parto ou puerpério. Nessa perspectiva, a etapa do histórico de enfermagem deve incluir a coleta de informações contextuais de qualidade, pois avaliações insuficientes ou inadequadas podem levar a diagnósticos e / ou prescrições inadequadas e julgamentos inadequados. Por outro lado, um histórico completo e informações relacionadas apoiarão intervenções de enfermagem mais eficazes e melhorarão a qualidade dos cuidados prestados às mães e bebês (ALMEIDA et al., 2018).

Segundo TELES et al. (2019), os Diagnóstico de Enfermagem para essas gestantes são Sobrecarga de estresse, Manutenção ineficaz da saúde, Comportamento de saúde 
propenso à risco, Obesidade, Risco de função cardiovascular prejudicada, Risco de binômio mãe-feto perturbado, Risco de infecção, Risco de sangramento, Conforto prejudicado, Ansiedade, Dor aguda e Náusea. Segundo GOMES (2016), os DE mais comuns são: Conhecimento deficiente, Risco de infecção, Dor aguda, Manutenção do lar prejudicada, Ansiedade, Medo, Manutenção ineficaz da saúde, Distúrbio no padrão de sono, dentre outros.

\section{PAPEL DO ENFERMEIRO NA ELABORAÇÃO DA SAE E PE}

A enfermagem, conforme descrito por Castro et al. (2016), é uma profissão que vive em constante mudança, desenvolvendo novos conhecimentos e qualificações profissionais, como conceitos básicos com fundamento em teorias elaboradas a partir das aprendizagens práticas hospitalares. No início da década de 50 a sistematização da assistência de enfermagem (SAE) foi discutida para a melhoria e dedicação ao importante documento do prezado cliente.

O enfermeiro com sua qualificação e capacidade tem garantindo um lugar para

ação, utilizando sua experiencia para uma assistência com planos e ideias que possa ajudar com o pré-natal dessa mulher. Nesse contexto, estudos demonstram a veracidade de que a consulta de enfermagem tem sido algo muito importante, porque pode assegurar uma melhor condição da assistência no pré-natal, através de prevenção e promoção, e um acompanhamento adequado a essas gestantes, mostrando assim o domínio técnico e humanística do profissional enfermeiro (TELES et al., 2019).

A atitude dos enfermeiros também tem um impacto significativo no processo de certificação hospitalar. Certificação para promover a qualificação dos serviços de saúde, com foco na segurança do paciente e no uso racional de ferramentas de apoio ao cuidado, aspecto intimamente relacionado ao processo de cuidar (DIAS et al., 2018).

Para os autores Castro et al. (2016), a SAE é um documento importante que visa anotar e priorizar o atendimento e a assistência ao cliente oferecendo apoio, segurança e cuidados, além de auxiliar e ajudar a organização do trabalho de enfermagem. Oferece um caminho para realizar ações de enfermagem, uma passagem de plantão mais prática, 
transformando o atendimento do cliente particularizado, caracterizado, completo e satisfatório.

Os enfermeiros participam e ajudam muito nessa etapa da vida da mulher, pois fazem um importante trabalho nesse momento sendo competente. Com seu conhecimento contribuem com a conservação do equilíbrio físico/psíquico da gestante e do recémnascido, sabendo identificar os períodos críticos em que suas intervenções são necessárias para que ambos fiquem bem. A enfermagem tem oportunidade de criar vínculo com a parturiente e promover um cuidado diferenciado e efetivo à mesma, através de uma assistência qualificada, acolhedora e humanizada (FRAGA et al., 2018).

Através da SAE, o enfermeiro põe em prática seus saberes técnico-científicos e humanos na assistência ao paciente tornando sua prática profissional, fortalecendo seu papel autonomicamente (CASTRO et al., 2016).

Apesar de a SAE ser privativo do enfermeiro, ela oferece uma estrutura organizada, planejamento e execução de ações sistematizadas, feita pelo pessoal de enfermagem durante o período que o paciente estiver sob os cuidados deles (CASTRO et al., 2016).

O enfermeiro necessita de uma metodologia correta para implementar a sistematização da assistência, no qual pode ser utilizada o PE. Essa ferramenta possui 5 partes no qual tem uma sequência e uma relação entre si, baseada no conceito de Wanda de Aguiar Horta (CASTRO et al., 2016).

Para garantir um bom cuidado de enfermagem individual, visando suas verdadeiras necessidades é preciso que este seja conduzido pelo Processo de Enfermagem (PE), baseado em Diagnósticos de Enfermagem (DE) (GOMES, 2016). Nesse entendimento, a assistência de enfermagem é de extrema importância em uma consulta de enfermagem de um pré-natal de uma gestante em alto risco, no qual o enfermeiro tem que dar prioridade à prevenção de complicações materno-fetal, encorajar para que a gestante faça do autocuidado, colocando em pratica a educação em saúde esclarecendo qualquer dúvida que surgir e orientar essa gestante (FELCZAK et al., 2018).

Segundo MEDEIROS et al. (2016), as intervenções para essas gestante são acomodar a paciente no leito e oferecer roupas adequadas; aferir e anotar sinais vitais de 
2/2 horas; apoiar a paciente em suas necessidades; comunicar ao enfermeiro alteração na saturação, frequência cardíaca e pressão arterial; lavar as mãos antes e após contato com a paciente; manter leito limpo e organizado; manter monitoração cardíaca contínua e oximetria de pulso; observar e registrar a amamentação; observar local de punção venosa; observar nível de consciência; promover mudança de decúbito de 2/2hs; realizar acesso venoso $\mathrm{s} / \mathrm{n}$; realizar higiene oral e corporal diariamente; realizar troca de acesso venoso periférico a cada 72 hs; realizar troca de equipo de drogas e, para Teles et al. (2019), uma boa assistência à essas mulheres podem mudar os prognósticos da mãe e do bebê, ajudando para que ocorra maior número de desfechos favoráveis.

\section{DISCUSSÃO}

De acordo com HORTA (1979), o ser humano possui necessidades básicas, no qual se divide em 3 eixos: necessidades psicobiológicas, psicossocial e psicoespiritual. Uma das principais necessidades psicobiológicas apresentadas pelos autores é aquela baseada em chegar o mais perto possível de adquirir a homeostasia da gestante, evitando tanto mãe quanto bebê vir a óbito.

Comunicação e aprendizagem foram as necessidades que os autores mais enfatizaram, pois, muitas pacientes não apresentam conhecimento sobre o problema e como se comportar perante a situação, então o enfermeiro utiliza a comunicação para auxiliar nesse processo para facilitar a assistência que a gestante receberá.

E por fim a fé foi a mais utilizada pelos autores em relação as necessidades psicoespiritual, pelo fato de quando ocorre o diagnóstico de eclampsia e ou pré-eclâmpsia a vida da mãe e do bebê corre risco, então, as mães utilizam a fé, no qual resulta em uma força, para passar por todo esse processo.

As mudanças fisiopatológicas que aparecem durante a gestação, sejam leves ou bem aparentes, se encontra entre as mais ressaltadas que o organismo humano sofre, gerando várias situações, apavoro, perguntas, apreensão, ansiedade e curiosidade. Nesse modo o enfermeiro vem sobressaindo como especialista eficaz para realizar as ações recomendado pela Organização Mundial da Saúde (OMS), em relação a assistência humanizada às 
grávidas, realizando um dever marcante pela promoção e prevenção da saúde, devendo ressaltar o entendimento e a realização da Sistematização da Assistência de Enfermagem (SAE) (BORBA et al., 2019).

Dessa forma, percebe-se que não somente as alterações fisiológicas decorrentes da gestação de alto risco se caracterizam em necessidades de assistência, mas também as alterações biopsicossociais e socioeconômicas devem ser foco da abordagem da equipe multidisciplinar no atendimento da gestante, cabendo ao enfermeiro o acolhimento, a escuta e ações humanísticas para atender essas necessidades e melhor assistir à gestante nesse seu processo de saúde-doença. Tais ações devem ser incorporadas na assistência de enfermagem desde o início do pré-natal.

Nesse aspecto, entende-se que uma das necessidades de assistência de enfermagem se dá no processo de orientação à gestante, principalmente as gestantes de idade avançada por se caracterizar como gestação de alto risco, para que bem informadas possam perceber qualquer sinal que indique possível complicação e, procurar assistência imediata.

Além dos diagnósticos de enfermagem presentes no NANDA-I (2018-2020 elencados pelos autores supracitados, podemos perceber que outros diagnósticos presentes nessa mesma versão do NANDA-I, se encaixam perfeitamente bem para atender às necessidades biopsicossociais e espirituais de gestantes com eclâmpsia e ou pré-eclâmpsia, sendo eles: Débito cardíaco diminuído (Volume de sangue bombeado pelo coração inadequado para atender às demandas metabólicas do organismo); Volume de líquidos excessivo (Entrada excessiva e/ou retenção de líquidos); Autonegligência (Conjunto de comportamentos culturalmente estruturados que envolvem uma ou mais atividades de autocuidado em que há falha em manter um padrão de saúde e bem-estar socialmente aceito); Risco de desequilíbrio eletrolítico (Suscetibilidade a mudanças nos níveis de eletrólitos séricos que pode comprometer a saúde)e, Fadiga (Sensação opressiva e prolongada de exaustão e capacidade diminuída de realizar trabalho físico e mental no nível habitual). 


\section{CONSIDERAÇÕES FINAIS}

A pesquisa evidenciou que gestantes com eclâmpsia e/ou pré-eclâmpsia correm um grande risco de vir a óbito, não somente elas como também seus bebês. A eclampsia pode aparecer a qualquer momento, podendo ser na gravidez, parto ou após o parto. A doença é uma complicação grave da gravidez que, se não for tratada imediatamente, poderá acarretar complicações graves e até fatais para mães e bebês. Às vezes, não há sintomas. Pressão alta e proteína na urina são as principais características da doença.

Para uma melhor assistência à gestantes com pré-eclâmpsia ou eclâmpsia o enfermeiro deve aplicar a SAE, de forma individualizada, com qualidade, com assistência integral e humanizada, focada nas necessidades biopsicossociais e espirituais, principalmente durante todo o pré-natal. Além da SAE, o enfermeiro deve inserir essas gestantes numa assistência com abordagem multidisciplinar para a integralidade da assistência de forma a atender às suas necessidades de forma holística.

Conclui-se que, o papel do enfermeiro na aplicabilidade da SAE é de suma importância e que, quando realizada de forma adequada, a SAE permite ao enfermeiro a identificação precoce de riscos ou alterações que esta gestante esteja sofrendo, com indício de pré-eclâmpsia ou eclâmpsia, na qual pode se ter um desfecho favorável, evitando a ocorrência de óbito materno-fetal. Desta forma, a SAE pode instrumentalizar o enfermeiro para uma abordagem assistencial, direcionando cuidados específicos para essas pacientes, evitando-se resultados negativos.

Esse estudo procurou contribuir para uma visão voltada para a Sistematização da Assistência de Enfermagem para gestantes com eclâmpsia e/ou pré-eclâmpsia estimulando que o enfermeiro promova um cuidado de qualidade e peculiar, de forma clara e organizada, direcionado os cuidados às necessidades da cliente.

\section{REFERÊNCIAS}

AGUIAR, M. I. F.; FREIRE, P. B. G.; CRUZ, I. M. P.; et al. Sistematização da Assistência de Enfermagem a paciente com síndrome hipertensiva específica da gestação. Revista da 
Rede de Enfermagem do Nordeste, v. II, n. 4, p. 66-75, out./dez.2010. Disponível em < http://www.periodicos.ufc.br/rene/article/view/46oo>. Acessado em: 09 mar. 202I.

ALDRIGHI, J. D.; RIBEIRO, S. da S.; CHEMIM, A. K.; WALL, M. L.; ZUGE, S. S.; PILER, A. A. Ocorrência de complicações no período gestacional em mulheres com idade materna avançada. Revista Baiana de Enfermagem, [S. 1.], v. 35, 2021. DOI: 10.1847I/rbe. v35.43083. https://periodicos.ufba.br/index.php/enfermagem/article/view/43083. Acesso em: is out. 2021.

ALMEIDA, V. S.; QUERIDO, D. L.; ESTEVES, A. P. V. S.; et al. Validação de instrumento para histórico de enfermagem materno-infantil utilizando Horta: estudo metodológico. Online Brazilian Journal of Nursing, v.17, n.I, p. 28-42, mar. 2018. ilus, tab. Disponível em 〈http://www.objnursing.uff.br/index.php/nursing/article/view/5858/html.$\quad$ Acessado em or jun. 202I.

AlveS, F. L. C.; SOUZA, F. K. R.; LIRA, M. C. P. S.; RODRIGUES, F. L. S.; et al. Grupo de gestantes de alto-risco como estratégia de educação em saúde. Revista Gaúcha de Enfermagem, v. 40, n. 40:e20180023, 2019. Disponível em: https://www.scielo.br/j/rgenf/a/STgFwJs6TLfstfsjxxG ${ }_{3} P Q N /$ ?format=pdf\&lang=pt

ANTUNES, M. B.; DEMitTo, M. O.; GRAVENA, A. A. F.; et al. Síndrome hipertensiva e resultados perinatais em gestação de alto risco. Revista da Rede de Enfermagem do Nordeste, v. 2I p. I-6, 2017. Tab. Disponível em: $\langle$ https://cdn.publisher.gni.link/reme.org.br/pdf/eio57.pdf〉. Acessado em or jun. 202I ARES, L. P. M. et al. Tecnologias não invasivas na assistência às parturientes de alto risco: percepções de enfermeiras obstétricas. Revista da Rede de Enfermagem do Nordeste, v. 22, e61385, 2021. Disponível em: www.periodicos.ufc.br/rene. Acesso em: 23 abr. 2021. 
BELARMINO, A. C.; HOLANDA, L. C. A.; FERREIRA, A. R. Convergência da síndrome de Vogt-Koyanagi-Harada e pré-eclâmpsia. Revista de Enfermagem Universidade Federal de Pernambuco on line, [S.1.], v. I2, n. Io, p. 2775-2783, out. 2018. ISSN $198 \mathrm{I}-8963$.

Disponível em: 〈https://periodicos.ufpe.br/revistas/revistaenfermagem/article/view/23586o/30250〉. Acesso em: is out. 2021.

BORBA, A. M.; SANTOS, A. B. R.; FERRAZ, A. C. D.; et al. Aplicação da sistematização da assistência de enfermagem em gestantes atendidas no pré-natal. Revista Ciência Plural; v. $\quad 5, \quad$ n. 3 , p. 89-102, 2019. Tab. Disponível em $\langle$ https://periodicos.ufrn.br/rcp/article/view/r8713/12235〉. Acessado em: I8 mar. 2021.

CASSIANO, A. N.; VITORINO, A. B. F.; SILVA, M. L. C.; et al. Desfechos perinatais de gestantes com pré-eclampsia grave: Estudo transversal. Online Brazilian Journal of Nursing; v.I8, n.4, dez. 2019. Tab. Disponível em: $\langle$ http://www.objnursing.uff.br/index.php/nursing/article/view/6205/html_2〉. Acessado em or jun. 2021

CASTRO, R. R.; AlVINO, A. L. F. N.; ROUBERTE, E. S. C.; et al. Compreensões e desafios acerca da sistematização da assistência de enfermagem. Revista de enfermagem da Universidade do Estado do Rio de Janeiro; v. 24, n.5: eio46I, set./out. 2016. Disponível em < http://dx.doi.org/Io.12957/reuerj.2016.1046I>. Acessado em or jun. 202I

COFEN. Conselho Federal de Enfermagem. Resolução no 358, de is de outubro de 2009. Dispõe sobre a Sistematização da Assistência de Enfermagem e a implementação do Processo de Enfermagem em ambientes, públicos ou privados, em que ocorre o cuidado profissional de Enfermagem, e dá outras providências. Brasília. 2009. Disponível em: 〈http://www.cofen.gov.br/resoluo-cofen-3582009_4384.html>. Acessado em: 28 mar. 2021. 
DIAS, L. B.; DURAN, E. C. M. Atitudes dos enfermeiros frente ao Processo de Enfermagem de um hospital público: estudo descritivo. Revista de enfermagem da Universidade do Estado do Rio de Janeiro, Rio de Janeiro, 2018; v. 26 :e26412. Disponível em < : http://dx.doi.org/10.12957/reuerj.2018.26412 >. Acessado em or jun. 2021.

DUleY L.; GÜlmeZOGLU, A. M.; et al. Magnesium sulphate and other anticonvulsivants for women with pre-eclampsia. Cochrane Database Systematic Reviews; v.II, CDoooo25, 2010. Acessado em or jun. 2021.

FELCZAK, C.; RAVELli, A. P. X.; SKUPIEN, S. V.; et al. Perfil de gestantes cardiopatas: alto risco. Revista Cogitare Enfermagem; v.23, n.2: e49605, 2018. Disponível em: <

https://docs.bvsalud.org/biblioref/2018/o6/885155/49605-233960-I-pb.pdf>. Acessado em or jun. 2021

FERREIRA, E. T. M.; MOURA, N. S.; GOMES, M. L. S.; et al. Características maternas e fatores de risco para pré-eclâmpsia em gestantes. Revista da Rede de Enfermagem do Nordeste; v. 20, n.I: e40327, jan.-dez. 2019. Disponível em:< http://periodicos.ufc.br/rene/article/view/40327/pdf $>$. Acessado em or jul. 2021

FERREIRA, M. B. G.; SILVEIRA, C. F.; SILVA, S. R.; et al. Assistência de enfermagem a mulheres com pré-eclâmpsia e/ou eclâmpsia: revisão integrativa. Revista da Escola de Enfermagem da Universidade de São Paulo; 2016; v. 5o, n. 2, p.324-334. Disponível em $\quad$ https://www.scielo.br/scielo.php?pid=Soo8o62342016000200324\&script=sci_arttext\&tlng=pt $>$. Acessado em: 09 mar. 202I.

FRAGA, T. F.; et al. Processo de enfermagem em centro obstétrico: perspectiva dos enfermeiros. Artigo extraído da dissertação - Processo de Enfermagem para o cuidado 
humanizado à parturiente de risco habitual em Centro Obstétrico, apresentada ao Programa de Pós-Graduação em Gestão do Cuidado em Enfermagem da Universidade Federal de Santa Catarina (UFSC), em 2016. Texto \& Contexto - Enfermagem [online]. 2018, v. 27, n. 3 [Acessado I5 Out. 2021], e46ooor6. Disponível em: 〈https://doi.org/10.1590/o104-070720180004600o16〉.

GOMES, L. F. S. Sistematização da assistência de enfermagem à gestante de alto risco: construção e validação de uma tecnologia para o cuidado. 2016. 200 f. Tese (Doutorado em Enfermagem) - Faculdade de Farmácia, Odontologia e Enfermagem, Universidade Federal do Ceará, Fortaleza, 2oi6. Disponível em: http://www.repositorio.ufc.br/bitstream/riufc/22135/r/2016_tese_lfsgomes.pdf . Acessado em I8 mar. de 202I

GONÇALVES, G. A.; PAES, L. B. O.; PARRO, M. C.; et al. Aspectos sociodemográfico, clínico-obstétrico e laboratorial na síndrome hipertensiva na gravidez. Revista CuidArte.

Enfermagem, v. I3, n. I, pp 27-31, jan. 2019. Disponível em: http://www.webfipa.net/facfipa/ner/sumarios/cuidarte/2019vi/27.pdf. Acesso em: is out. 2021.

HORTA, Wanda de Aguiar. Processo de Enfermagem. Wanda de Aguiar Horta, com a colaboração de Brigitta E. P. Castellanos. - São Paulo: EPU 1979.

JORGE, H. M. F.; SILVA, R. M.; MAKUCH, M. Y. Humanized care in high-risk prenatal care: nurses' perceptions. Revista da Rede de Enfermagem do Nordeste. 2020; v.21 :e4452I. Disponível em: <https:// doi.org/10.15253/2175-6783.20202144521>. Acessado em 23 out. 2021

JUNIOR, A. R. F.; OlIVEIRA FILHO, J. T.; RODRIGUES, M. E. N. G.; et al. O enfermeiro no pré-natal de alto risco: papel profissional. Revista Baiana de Saúde Pública, 
v. 4I, n. 3, pp. 650-667, jul./set. 2017. Disponível em: https://rbsp.sesab.ba.gov.br/index.php/rbsp/article/view/2524. Acesso em: I4 out. 202I

LIMA, B. C. S.; RIBEIRO, M. M. A.; MARTINS, E. R. C.; et al. Sentimentos de gestantes de risco durante a fase de indução: estudo descritivo. Online Brazilian Journal of Nursing, v. 15, n. 2, pp. 254-264, jun. 2016.

MACHADO, J. P. C.; SILVA, D. M.; SOUZA, E.; et al. Percepção de enfermeiros de unidades de internação clínica sobre a Sistematização da Assistência de Enfermagem. Revista Nursing; v. 22, n. 257, p. 3220-3225, out.2019. Disponível em 〈http://www.revistanursing.com.br/revistas/257/pg50.pdf〉. Acessado em: I8 mar. 2021.

MEDEIROS, A. L.; SANTOS, S. R.; CABRAL, R. W. L.; et al. Avaliando diagnósticos e intervenções de enfermagem no trabalho de parto e na gestação de risco. Revista Gaúcha de Enfermagem. 2016 set; v. 37 , n.3 :e55316. Disponível em: $<$ http://www.revenf.bvs.br/scielo.php?script=sci_arttext\&pid=Si983-14472016000300409>. Acessado em or jun. 202I

MELO, B. C. P.; AMORIM, M.M.R.; KATZ, L. et al. Perfil epidemiológico e evolução clínica pós-parto na pré- eclampsia grave. Revista da Associação Médica Brasileira; 2009; v. 55, n. $\quad$ 2, $\quad$ p. $\quad$ 175-80. $\quad$ Disponível em $\langle$ https://www.scielo.br/scielo.php?script=sci_arttext\&pid=So104-42302009000200022〉. Acessado em 29 abr. 2021.

MELO, M. N. et al. Cuidado hospitalar de mulheres que vivenciaram a gestação de alto risco: contribuições para a enfermagem. Revista de Enfermagem Universidade Federal de Pernambuco, [S.1.], v. io, n. II, p. 3911-3917, ago. 2016. ISSN 1981-8963. Disponível em: 〈https://periodicos.ufpe.br/revistas/revistaenfermagem/article/view/II472〉. Acesso em: I5 out. 2021. doi:https://doi.org/ro.5205/1981-8963-vioiriaII472p3911-3917-2016. 
MOLA, R.; DIAS, M. L.; COSTA, J. F. et al. Conhecimento dos profissionais de enfermagem sobre a sistematização da assistência de enfermagem. Revista de Pesquisa Cuidado é Fundamental; v. II, n. 4, p. 887-893, jul.-set. 2019. Tab. Disponível em $\langle$ http://www.seer.unirio.br/index.php/cuidadofundamental/article/view/67oo/pdf_I . Acessado em: I8 mar. 2021.

NANDA. Diagnósticos de enfermagem da NANDA-I: definições e classificação 2018-2020 [recurso eletrônico] / [NANDA International]; tradução: Regina Machado Garcez; revisão técnica: Alba Lucia Bottura Leite de Barros... [et al.]. - II. ed. - Porto Alegre: Artmed.

NIC. Classificação das intervenções de enfermagem (NIC) / Gloria M. Bulechek, Howard K. Butcher, Joanne McCloskey Dochterman; [tradução Soraya Imon de Oliveira... et al]. - Rio de Janeiro: Elsevier, 2010

NÓBREGA, M. F. et al. Perfil de gestantes com síndrome hipertensiva em uma maternidade pública. Revista de Enfermagem Universidade Federal de Pernambuco, [S.1.], v. Io, n. 5, p. I805-18II, abr. 2016. ISSN I98I-8963. Disponível em: 〈https://periodicos.ufpe.br/revistas/revistaenfermagem/article/view/1356o〉. Acesso em: I5 out. 2021.

NOC. Classificação dos resultados de enfermagem (NOC)/Sue Moorhead... [et al.]; [tradução Regina Machado Garcez... et al]. - Rio de Janeiro: Elsevier, 2010.

OLIVEIRA, G. S. et al. Assistência de enfermeiros na síndrome hipertensiva gestacional em hospital de baixo risco obstétrico. Revista Cuidarte, v. 8, n. 2, p. 156I1572, Dec. 2017. Available from

〈http://www.scielo.org.co/scielo.php?script=sci_arttext\&pid=S22160973201700020156I\&lng =en $\& \mathrm{nrm}=$ iso $>$. access on I5 out. 2021. 
ORCY, B.; PEDRINI, R.; PICCININI, P. et al. Diagnóstico, fatores de risco e patogênese da Pré-eclâmpsia. Revista Hospital de Clínicas de Porto Alegre; 2007; v. 27, n.3. Disponível em < https://lume.ufrgs.br/handle/10183/28917>. Acessado em 29 abr. 202I.

PERAÇOLI, J. C.; BORGES, V. T. M.; RAMOS, J. G. L.; et al. Pré-eclâmpsia/eclâmpsia. Revista Feminina 2019, v. 47, n. 5, p. 258-273, 0531. ilus, tab. Disponível em: 〈https://docs.bvsalud.org/biblioref/2020/o7/1046517/femina-2019-475-258-273.pdf〉. Acessado em or jun. 2021

PERAÇOLI, J. C. et al. Síndrome HELLP recorrente: relato de dois casos. Revista Brasileira de Ginecologia e Obstetrícia. 1998, v. 20, n. 3, pp. 165-167. Disponível em: 〈https://doi.org/10.1590/Soroo-72031998000300008〉. Acessado I5 out. 2021.

POZZA, L. V.; DELANEY, L. A.; BURMANN, L. et al. Crise hipertensiva gestacional. Revista Acta Médica, v. 37, n.5, 2016. Disponível em:<https://docs.bvsalud.org/biblioref/2018/o4/882968/31-crise-hipertensivagestacional.pdf $>$. Acessado em or jun.202I

ROCHA; L.; MELO, C.; COSTA, R.; et al. A comunicação de más notícias pelo enfermeiro no cenário do cuidado obstétrico. Revista Mineira de Enfermagem, v. 20:e98I, 20r6. Disponível em: 〈https://cdn.publisher.gnı.link/reme.org.br/pdf/e98I.pdf 〉. Acessado em or jun. 2021.

SILVA, P. L. N.; OLIVEIRA, J. S.; SANTOS, A. P. O.; et al. Cuidados pré-natais e puerperais às gestantes de um centro de saúde de Minas Gerais quanto ao risco de préeclâmpsia: aspectos clínicos, nutricionais e terapêuticos. Journal of Health \& Biological Sciences., v.5, n.4, pp. 346-35I, 2017. Disponível em < https://periodicos.unichristus.edu.br/jhbs/article/view/1222/48I >. Acessado em or jun. 2021. 
SOUZA, B. F. et al. Enfermagem e gestantes de alto risco hospitalizadas: desafios para integralidade do cuidado. Programa de Pós-Graduação em Enfermagem, Universidade Federal de São Carlos, 2017. Revista da Escola de Enfermagem da Universidade de São Paulo [online]. 2020, v. 54 e03557. Disponível em: 〈https://doi.org/ro.159o/Sig8o220X2018036903557>. Acesso em I5 out. 2021.

SOUZA, M.; SILVA, M. D.; CARVALHO, R. Revisão integrativa: o que é e como fazer. $\begin{array}{lllllll}\text { Revista } & \text { Einstein } 2010 ; \quad \text { v.8, n.I, p.Io2-6. Disponível em }\end{array}$ 〈https://www.scielo.br/j/eins/a/ZQTBkVJZqcWrTT34cXLjtBx/?format=pdf\&lang=pt > . Acessado em 28 mai. 2021. Acessado em i5 out. 2021.

TAVARES, D. S. et al. CONSTRUÇÃO E VALIDAÇÃO DE UM HISTÓRICO DE ENFERMAGEM PARA CONSULTA PRÉ-NATAL. Revista Enfermagem em Foco, [S.1.], v. Io, n. 7, p. 2357-707, fev. 2020. ISSN X. Disponível em: $\langle$ http://revista.cofen.gov.br/index.php/enfermagem/article/view/2333/547〉. Acesso em: I6 out. 2021. Acessado em I5 out. 202I.

TEleS, P. A.; COSTA, E. M.; PANOBIANCO, M. S.; et al. Diagnósticos de Enfermagem mais prevalentes em gestantes de alto risco. Revista Enfermagem em Foco 2019; v. Io, $\quad$ n. $\quad 3, \quad$ p. II9-I25. $\quad$ Disponível em $\langle$ http://revista.cofen.gov.br/index.php/enfermagem/article/view/1937/571 $>$. Acessado em I8 mar. 202I.

THUlER, A. C. M. C.; WALL, M. L.; BENEDET, D. C. F.; et al. Medidas preventivas das síndromes hipertensivas da gravidez na atenção primária. Revista Enfermagem Universidade Federal de Pernambuco., Recife, v. 12, n. 4, pp. 1060-71, abr. 2018. Disponível em 〈https://periodicos.ufpe.br/revistas/revistaenfermagem/article/view/234605/28678>. Acessado em I5 out. 2021. 
URSI, E. S. Prevenção de Lesões de Pele no Perioperatório: revisão integrativa da literatura. Dissertação. [Mestrado em Enfermagem]. Escola de Enfermagem de Ribeirão Preto da Universidade de São Paulo. Ribeirão Preto. 130 p. 2005. Disponível em $:<$ https://www.teses.usp.br/teses/disponiveis/22/22132/tde-18072005095456/publico/URSI_ES.pdf>.

VILlALBA, Jessica Paola Garcia. Processo assistencial das mulheres com morbidade materna grave: um estudo misto. 2019. 98 f. Dissertação (Mestrado em Enfermagem) Universidade do Estado do Rio de Janeiro, Rio de Janeiro, 2019. Disponível em: https://www.bdtd.uerj.br:8443/handle/r/III98. Acesso em: I5 out. 2021. 